\title{
Advanced Adverse Outcome Pathways Potentially Bridging Pathogenesis of COVID-19
}

\author{
Young Jun Kim ${ }^{1,2 *}$, Chang Gyun Park ${ }^{1}$, Sang Rak Lim ${ }^{3}$ In Dong Jun and Yong Oh Lee ${ }^{3}$ \\ 1 Environmental Safety Group, Korea Institute of Science and Technology Europe Forschungsgesellschaft \\ $\mathrm{mbH}, 66123$ Saarbrücken, Germany \\ 2 Division of Energy \& Environment Technology, University of Science and Technology, Daejeon, Republic of \\ Korea 34113 \\ 3 Smart Convergence Group, Korea Institute of Science and Technology Europe Forschungsgesellschaft mbH, \\ 66123 Saarbrücken, Germany \\ * Correspondence: Young Jun Kim; E-mail address: youngjunkim@kist-europe.de; Tel: +49-681-9382-327; Fax: \\ +49-681-9382-319
}

\begin{abstract}
Increasing global concern over COVID-19 has recently brought greater attention to studies due to the ease of person-to-person transmission and the current lack of effective antiviral therapy. Here, we proposed the application of the adverse outcome pathway (AOP) framework to support research on the pathogenesis of viral disease. We first constructed adverse outcome pathways (AOPs) applicable to COVID-19 management to understand whether the infection causes severe acute respiratory distress. Based on the AOP framework where mechanistic elucidation of the pathway from the interaction of chemicals (or viruses) to apical endpoints is represented, our COVID19 AOP indicated that the molecular initiating event (MIE) was angiotensin-converting enzyme 2 (ACE2) interaction, and the key events (KEs) were the increased pro-inflammatory cytokines in immune cells, with increased mortality as an apical adverse outcome (AO). However, there is still limited information on the toxicity mechanisms of AOPs in COVID-19; therefore, detailed KEs and AOs on toxicity mechanisms will be required to fill these gaps in the data. This study demonstrated that the COVID-19 AOP framework is a suitable tool to design new drugs and to integrate crowdedsourced information for the battle against the COVID-19 pandemic.
\end{abstract}

Keywords: COVID-19; Angiotensin Converting Enzyme 2 (ACE2); Adverse outcome pathway (AOP); Molecular initiation Event (MIE); Key event (KE); Adverse Outcome (AO)

\section{Introduction}

Coronaviruses have caused severe and fatal respiratory tract infections in humans. The separate emergences of severe acute respiratory syndrome coronavirus (SARS-CoV) and coronavirus disease 2019 (COVID-19) have emphasized that the interaction between the virus and host should be elucidated for developing treatments and vaccines. COVID19 emerged with clinical presentations consistent with viral pneumonia, and the infection caused severe respiratory distress syndrome similar to SARS-CoV [1-3]. COVID-19, which has a genome sequence similar to that of SARS-CoV, is a novel type of coronavirus owing to a high degree of variation in the sequence of the $S$ glycoprotein $[1,4-6]$. Clinical data from Wuhan, China, showed multiorgan dysfunction, namely, acute respiratory distress syndrome (ARDS) [7-10]. In addition, the most severe sequela of pathogenic coronavirus infection-induced SARS is lung fibrosis; nearly $45 \%$ of SARS patients experience lung fibrosis after 3-6 months, which potentially sets an essential context for COVID-19 [11-15]. The high potential for re-emergence of a similar virus infection should be contemplated. Here, we proposed the application of the adverse outcome pathway (AOP) framework to support research on the pathogenesis of viral disease. Crowdsourcing towards the establishment of COVID-19 AOPs is organized by the European Commission's Joint Research Centre (JRC) (www.ciao-covid.net). An AOP endorsed by OECD was initially developed 
as knowledge management for capturing and disseminating mechanistic information to support regulatory toxicology. An AOP is described as a series of changes in the measurable biological event and state linkage between a molecular initiating event (MIE) and key events (KEs) that triggers a perturbation and occurrence of an adverse outcome (AO) [1618]. The immediate application of the AOP framework needs to support the investigation and depiction of the mode of action of COVID-19 from the molecular to the population level, which has the potential to become the basis of a mechanistic understanding of virusinduced multiorgan injury and its remediation properties and further validation of drug candidates for use in practice. The underlying concept of AOP development provides practical use of mechanistic knowledge for the design and development of alternatives to animal testing. Moreover, quantitative analyses of key event relationships (KERs) and cross-species interpretation contribute to the study of a new epidemic disease based on mechanistic information from vast research fields of human diseases and medicine.

\section{Mechanistic understanding between COVID-19 infection}

Recently, we published a data fusion pipeline for enriching and refining AOP 320 based on molecular information, and biological pathway data were proposed [19], as shown in Figure 1. The coronavirus S (spike) glycoprotein employs ACE2 (angiotensinconverting enzyme 2) as a receptor for host cell entry. Binding of the coronavirus S glycoprotein to ACE2 subsequently triggers a conformational change in the S glycoprotein of the coronavirus, allowing for proteolytic digestion by host cell proteases (TMPRSS2), resulting in cell membrane fusion [20-22].

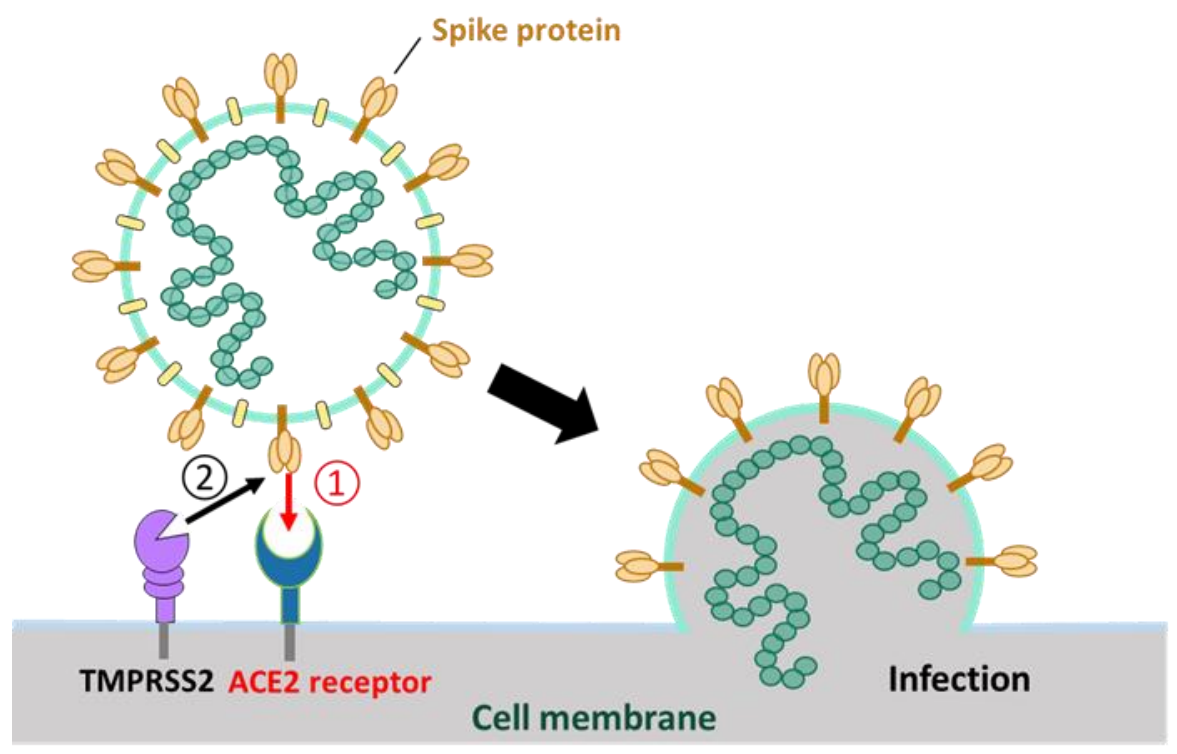

Figure 1. Common process of COVID-19 infection

The approach brings together information from curated life science databases and results in AOP-linked molecular descriptions of adverse outcomes supported by empirical data and bioinformatic analysis. Based on the concept proposed herein, to apply the AOP framework to explore COVID-19, the data fusion pipeline further detailed the pathogenesis.

\section{Adaptation to Adverse Outcome Pathways}

Further to enrich AOP 320 (Figure 2A), initial results detailing the molecular interactions in AOP 320 indicate substantial overlap with AOP 319, which describes substance interaction with ACE2 leading to lung fibrosis, as shown in Figure 2B [19]. Based on the concept proposed herein, to apply the AOP framework to explore COVID-19, there are 
two types of toxicity mechanisms. Initial events detail the viral S-protein perturbation in AOP320 and ACE2 downregulation in AOP 319. One of the significant MIEs in AOP320 is the binding of $S$ glycoprotein to the ACE2 receptor, leading to increased mortality. In the case of AOP319, lung fibrosis is triggered by inhibition of ACE2 protein catalytic activity. It remains possible that ACE2 down regulation is influenced by the interaction of the spike (S) protein with the ACE2 receptor [23].

A

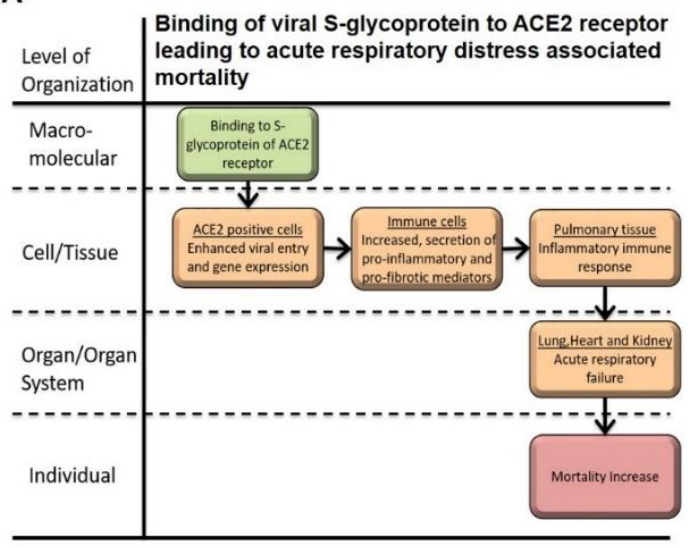

B

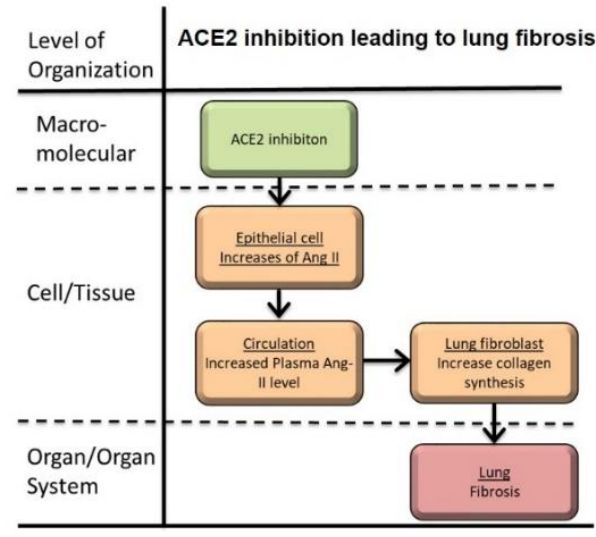

Figure 2. Schematic diagram of the putative AOPs for Covid 19. AOP 320 (A) and AOP 319 (B) in OECD AOP wiki (https://aopwiki.org/).

As shown in Figure 3, AOP 320 indicates that cell internalization of the ACE2/COVID-19 complex and viral replication trigger excess proinflammatory cytokine release by immune cells, which might play a major role in severe COVID-19 AO. These processes are most likely accompanied by NF-kB activation by COVID-19 proteins and IL-1 $\beta$ and IL-6 as KEs [24-26]. In particular, IL-6 has been reported to be dramatically increased in COVID-19 patients, with a strong implication in acute inflammation by activating JAK/STAT signaling and shedding ACE2 [24,27]. Finally, IL-6 contributes to excessive cytokine release, increasing the plasma levels of proinflammatory and profibrotic mediators. Similar to MIE in AOP 320, AOP 319 for lung fibrosis is characterized by ACE2 inhibition leading to excessive deposition of extracellular matrix (ECM) proteins, such as transforming growth factor-beta (TGF- $\beta$ ). However, the link between COVID-19 infection and lung fibrosis is still unclear. In our AOP 319 framework, we suggest the perturbation of the renin-angiotensin system in which Ang II accumulates by the interaction of COVID19 , which has undergone the downregulation of ACE2.

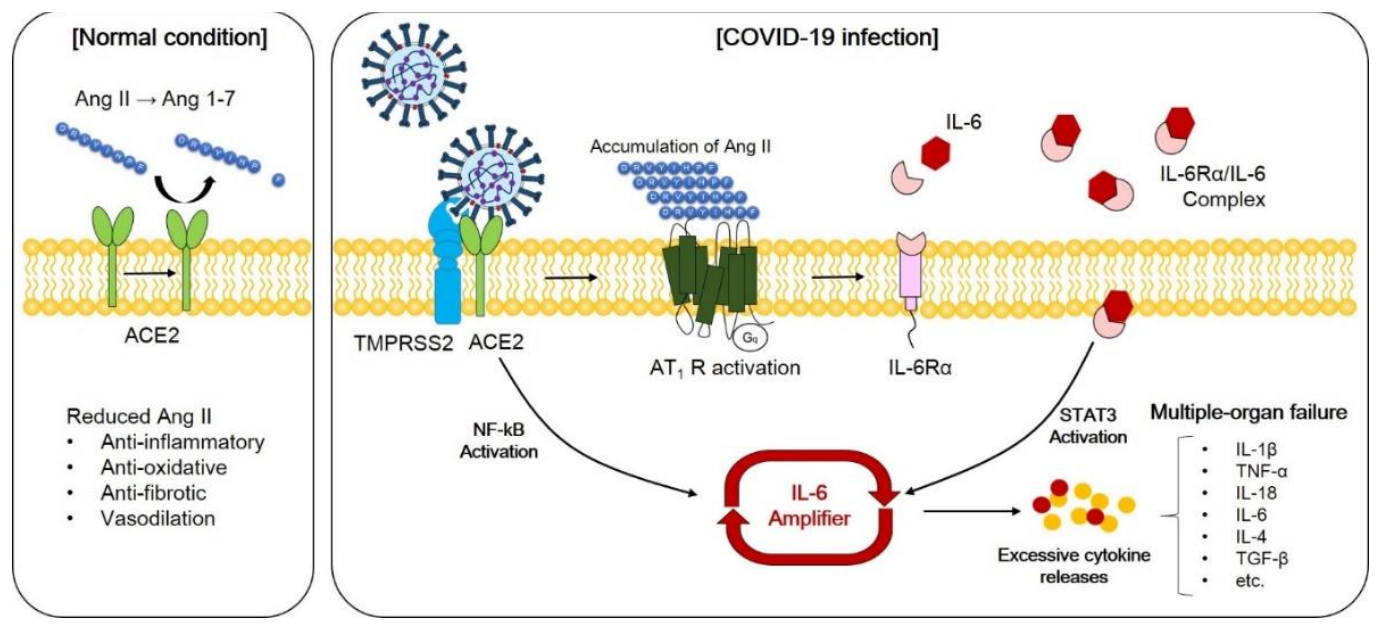


Figure 3. Simplified diagram of the renin-angiotensin system in normal and COVID-19 infection conditions.

The renin-angiotensin system (RAS) is a homeostatic regulator signalling pathway engaged in vascular function control. The regulation of blood pressure, vasoconstriction, vasodilation, and blood volume restore this signalling pathway [28]. Especially ACE2 is one of major enzyme of blood pressure regulation in the renin-angiotensin system. It is well known that angiotensin II (Ang II), a ligand for type I angiotensin receptor (AT1R), is the main effector peptide of the RAS and induces vasoconstriction, inflammation and fibrosis [29,30]. Figure 3 shows that ACE2 catalyzes Ang II to Ang (1-7), leading to vasodilation and anti-inflammatory and antifibrotic signals [31]. In the other way, ACE1 cleaves angiotensin I and generating angiotensin (Ang) II, which provokes vasoconstriction leading to the increases of vascular inflammation and fibrosis in patients infected with COVID-19. The effect of ACE1 signaling is by AT1R activation. ACE1 mediated AT1R functions as the critical mediator of Ang II actions and the counteract effects of ACE2, which is responsible for Ang II conversion to Ang (1-7) [32]. A higher irregularity between the action of ACE1 and ACE2 provokes ARD triggered by COVID 19. When COVID-19 is bound to its ACE2 receptor and TMPRSS2, ACE2 receptor down-regulation provokes an inhibitory effect of conversion from Ang I to Ang (1-7). Subsequently, the accumulation of Ang II binds to AT1R, resulting in IL-6 amplification, which triggers both inflammation and fibrosis. Therefore, ACE2 dysfunction associated with increased plasma IL-6 levels could be one of the tissue KEs for multi-organ failure via the activation of AT1R signaling.

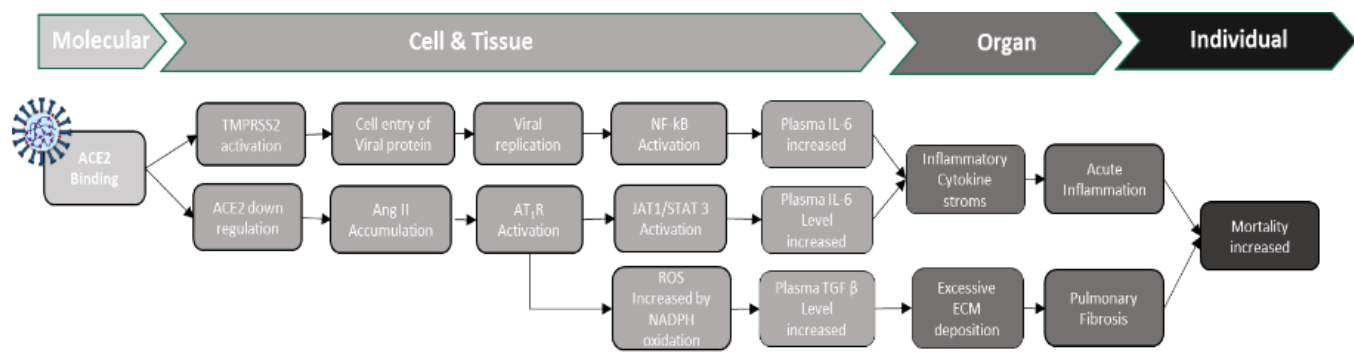

Figure 4. The network of AOPs of relevance to COVID-19.

As a result, we suggest that the AOP network that triggers immune responses by COVID-19 replication and Ang II accumulation by TMPRSS2 activation and ACE2 downregulation could lead to acute inflammation and pulmonary fibrosis, as shown in Figure 4. From the derived AOP network, it was observed that each event shares at least one common MIE and KEs. For instance, the MIE, defined as ACE2 binding, and increases in plasma IL-6 are shared in this AOP network. Gomolak et al. showed that IL-6 levels were almost 60-fold higher in mice treated with $1000 \mathrm{ng} / \mathrm{kg} / \mathrm{min}$ Ang II than in control mice regarding AOP319 [33]. We further provide a mechanistic link for AOP 320 that the increase in plasma IL-6 may relate to the level of Ang II accumulation caused by COVID-19 infections. IL-6 has been reported to be dramatically increased in COVID-19 patients, with a strong implication in acute inflammation and cytokine storm by activating JAK/STAT signaling and shedding ACE2 [24]. Ang II accumulation by ACE2 downregulation in AOP319 is questionable since it opens the possibility for the development of selective drugs that specifically target the ACE2 receptor domain used by the RBD of SARS-CoV-2 to enter the host cell without altering the production of Ang 1-7. An additional consideration concerns whether to include potential KEs from ROS synthesis to pulmonary fibrosis. It should be noted that Ang II promotes TGF- $\beta$-dependent fibrosis through ROS synthesis [31,34-38]. All these lines of evidence suggest that TGF- $\beta$ plays a crucial role in the development of fibrosis. Finally, our results indicate that COVID-19 binding to ACE2 
causes toxicity mainly through aberrant induction and imbalances of various pro-inflammatory cytokines and growth factors, ultimately leading to increased mortality. The binding of ACE2 leads to both acute inflammation and pulmonary fibrosis that is applicable to human health perspectives on COVID-19. However, more direct experimental evidence is still required to confirm not only the ACE2-RBD interaction but also the inter- and intracellular COVID-19 and human protein interactions.

\section{COVID 19 AOP application in drug discovery}

ACEIs/ARBs (Angiotensin-Converting Enzyme Inhibitors and Angiotensin Receptor Blockers), the powerful COVID 19 targeting the RAS, are used to treat a wide range of implications related to hypertension, cardiovascular disease, and renal disease. ACEIs functions not only lower blood pressure but also protect the pathogenesis of COVID-19 [39]. ACEIs/ARBs may prevent COVID-19 viral entry by stabilizing ACE2-AT1R complex and blocking viral protein ACE2 interaction and cell internalization. However, ACE2 activity is not directly influenced by ACEIs and the messenger RNA level of ACE2 increased with ACEI but was not observed with ARBs which did not show any association of mortality $[40,41]$. It is therefore suggested that the putative COVID-19 AOP network is applicable to viral infection management to understand the detailed molecular interactions that could comprise the different types of virus infections and replications, such as MERS-CoV and SARS-CoV, mediated by consensus ACE2 binding as MIE and KEs. For instance, antiRBD (MIE) combined with anti-inflammatory therapies (KEs) can be a promising treatment to reduce the risk of AO. The putative COVID-19 AOP network is applicable to viral infection management to understand toxicity mechanisms that could comprise the different types of virus infections and replications, such as MERS-CoV and SARS-CoV, mediated by consensus ACE2 binding as MIE and KEs. For instance, anti-RBD (MIE) combined with anti-inflammatory therapies (KEs) can be a promising treatment to reduce the risk of AO. Therefore, trials have identified chemicals that inhibit the binding between the ACE2 receptor and the spike protein by molecular docking [42-44]. For instance, dexamethasone, an anti-inflammatory drug, was recently considered for the treatment of severe cases of COVID-19 [45]. Based on the mode of action, Adewale Oluwaseun Fadaka et al. showed that the binding of dexamethasone to Mpro ( $-6.7 \mathrm{kcal} / \mathrm{mol})$, Glucocorticoid ( -14.3 $\mathrm{kcal} / \mathrm{mol})$ and IL-6 receptor $(-3.6 \mathrm{kcal} / \mathrm{mol})$ resulted in higher docking scores, which can attenuate pulmonary inflammation, thereby suppressing proinflammatory cytokines and causing lung damage as the key events [46]. Quite interestingly, the interactions of dexamethasone with S-RBD exhibited $-6.3 \mathrm{kcal} / \mathrm{mol}$ as the high binding energy, which shared some of the same interactive moieties as the ACE2 receptor with the S-RBD (spike-receptor binding domain), as described by MIE. The docking complex with dexamethasone exhibited a total of eleven interactions, comprising one hydrogen and one halogen (PHE490), two hydrophobic (LEU455, PHE490), and eight van der Waals interactions (LYS417, LYS458, PHE486, TYR489, PRO491, LEU492, GLN493, SER494), as shown in Figure 5. Previous studies identified the critical residues that interact with the ACE2 receptor, including LEU455, PHE486, GLN493, SER494, and ASN501 [4,5,47]. The residues of LEU455 and PHE486 in the S-RBD have been identified to interact with the ACE2 receptor domain $[4,5]$. In light of these facts, dexamethasone can also be screened by the MIE component to inhibit the interaction between ACE2 and S-RBD. 

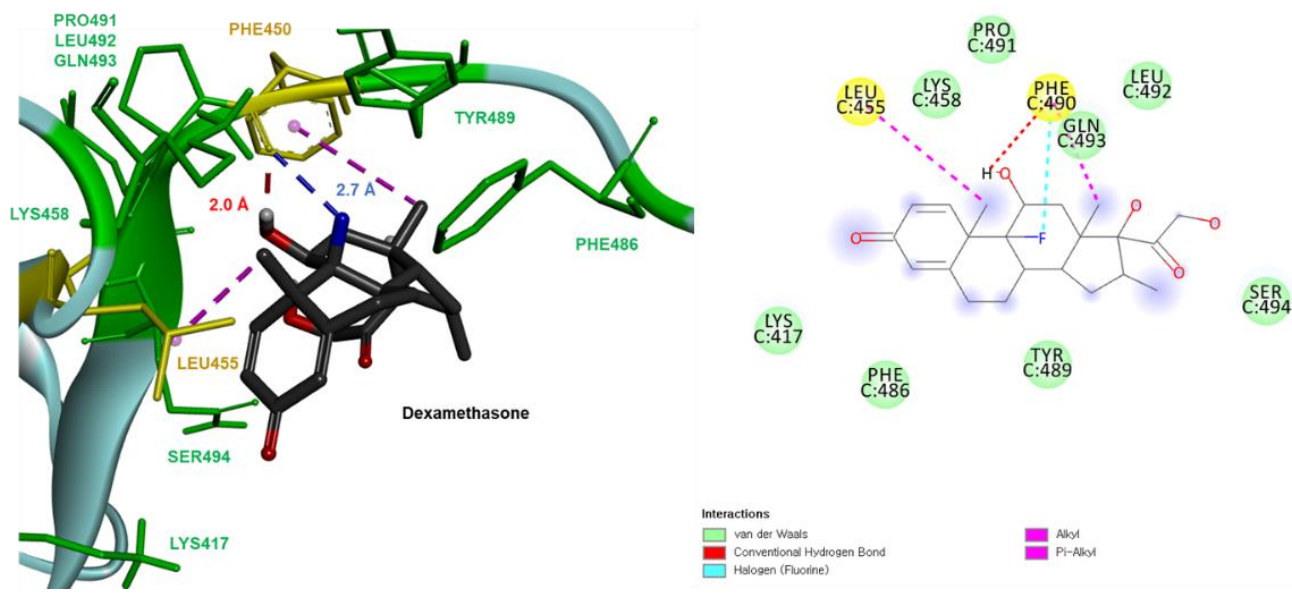

Figure 5. Representative molecular docking images of dexamethasone with the receptor binding domain of spike glycoprotein SARS-CoV-2 (S-RBD). The green color of residues indicates van der Waals interaction with the dexamethasone in 3D and 2D representation. The yellow color of residues indicates hydrogen, halogen, or hydrophobic interactions with the dexamethasone. The dotted lines of red, blue, and purple colors show hydrogen, halogen, and hydrophobic interactions respectively.

The overall COVID-19 AOP network could serve many purposes, a noticeable one being drug design targeting the interaction between ACE2 and S-RBD. In this regard, alternatives to animal testing can be engaged to support animal-free clinical decisions that allow prediction of particular aspects of COVID-19 infection. By mechanistically linking the AOP network, further insight into COVID-19 pathogenesis will be gained and measurable at the different organization levels in the host, which in turn will open perspectives for designing strategies to diagnose disease severity. However, there is limited evidence concerning the relationship between KEs. These preliminary results indicate the value of information curation and data integration, where AOPs play a central role in providing a transparent and user-friendly platform for collaboration across diverse fields. The benefits of bridging these fields extend in the parallel development of preventive and therapeutic medicine.

\section{Conclusions}

Taken together, AOPs provide a platform for collecting and categorizing data for a better understanding of underlying mechanisms for the entry of virus into cells mediated by ACE2. The importance of the structural spike (S) protein is remarkable due to its key role in COVID-19 entry through its interaction with the host receptor, ACE2. Using the AOP framework, we have shown the mechanistic pathway links between COVID-19 binding with ACE2 and adverse outcomes. These adverse outcomes may vary, such as fibrosis, thrombosis and inflammation, in COVID-19 patients. Thus, routine analysis and early prevention, diagnosis and treatment may be beneficial for COVID-19 patients. Overall, this study suggests that the AOP framework is a suitable tool to identify data gaps and to enable the development of preventive and therapeutic medicine in viral infection-associated diseases.

Author Contributions: Lim SR, Lee Y.O, Park CG, Jun ID and Kim YJ conceived AOP framework. Lim SR, Lee Y.O, Park CG, Jun ID and Kim YJ, wrote the manuscript. All authors read and approved the final manuscript.

Acknowledgments: This research was supported by the National Research Council of Science \&Technology (NST) grant by the Korea government (MSIP) (No. CAP-17-01-KIST Europe), and part of CIAO (Modelling the pathogenesis of COVID-19 using the Adverse 
Outcome Pathway framework) project in Joint Research Centre-European Commission (JRC-EC).

Conflicts of Interest: The author declares that he has no conflict of interest.

\section{References}

1. Hu, B.; Guo, H.; Zhou, P.; Shi, Z.-L. Characteristics of SARS-CoV-2 and COVID-19. Nature Reviews Microbiology 2020, 10.1038/s41579-020-00459-7, doi:10.1038/s41579-020-00459-7.

2. Zhu, N.; Zhang, D.; Wang, W.; Li, X.; Yang, B.; Song, J.; Zhao, X.; Huang, B.; Shi, W.; Lu, R., et al. A Novel Coronavirus from Patients with Pneumonia in China, 2019. New England Journal of Medicine 2020, 382, 727-733, doi:10.1056/NEJMoa2001017.

3. Nishiura, H.; Linton, N.M.; Akhmetzhanov, A.R. Initial Cluster of Novel Coronavirus (2019-nCoV) Infections in Wuhan, China Is Consistent with Substantial Human-to-Human Transmission. J Clin Med 2020, 9, 488, doi:10.3390/jcm9020488.

4. Shang, J.; Ye, G.; Shi, K.; Wan, Y.; Luo, C.; Aihara, H.; Geng, Q.; Auerbach, A.; Li, F. Structural basis of receptor recognition by SARS-CoV-2. Nature 2020, 581, 221-224, doi:10.1038/s41586-020-2179-y.

5. Wan, Y.; Shang, J.; Graham, R.; Baric, R.S.; Li, F. Receptor Recognition by the Novel Coronavirus from Wuhan: an Analysis Based on Decade-Long Structural Studies of SARS Coronavirus. Journal of Virology 2020, 94, e0012700120, doi:10.1128/jvi.00127-20.

6. Kumar, S.; Maurya, V.K.; Prasad, A.K.; Bhatt, M.L.B.; Saxena, S.K. Structural, glycosylation and antigenic variation between 2019 novel coronavirus (2019-nCoV) and SARS coronavirus (SARS-CoV). VirusDisease 2020, 31, 13-21, doi:10.1007/s13337-020-00571-5.

7. Renu, K.; Prasanna, P.L.; Valsala Gopalakrishnan, A. Coronaviruses pathogenesis, comorbidities and multiorgan damage - A review. Life Sci 2020, 255, 117839-117839, doi:10.1016/j.lfs.2020.117839.

8. Huang, C.; Wang, Y.; Li, X.; Ren, L.; Zhao, J.; Hu, Y.; Zhang, L.; Fan, G.; Xu, J.; Gu, X., et al. Clinical features of patients infected with 2019 novel coronavirus in Wuhan, China. The Lancet 2020, 395, 497-506, doi:10.1016/S01406736(20)30183-5.

9. Wu, C.; Chen, X.; Cai, Y.; Xia, J.a.; Zhou, X.; Xu, S.; Huang, H.; Zhang, L.; Zhou, X.; Du, C., et al. Risk Factors Associated With Acute Respiratory Distress Syndrome and Death in Patients With Coronavirus Disease 2019 Pneumonia in Wuhan, China. JAMA Internal Medicine 2020, 180, 934-943, doi:10.1001/jamainternmed.2020.0994.

10. Tian, J.; Yuan, X.; Xiao, J.; Zhong, Q.; Yang, C.; Liu, B.; Cai, Y.; Lu, Z.; Wang, J.; Wang, Y., et al. Clinical characteristics and risk factors associated with COVID-19 disease severity in patients with cancer in Wuhan, China: a multicentre, retrospective, cohort study. The Lancet Oncology 2020, 21, 893-903, doi:10.1016/S14702045(20)30309-0.

11. Xie, L.; Liu, Y.; Xiao, Y.; Tian, Q.; Fan, B.; Zhao, H.; Chen, W. Follow-up study on pulmonary function and lung radiographic changes in rehabilitating severe acute respiratory syndrome patients after discharge. Chest 2005, 127, 2119-2124, doi:10.1378/chest.127.6.2119. 
12. Hui, D.S.; Joynt, G.M.; Wong, K.T.; Gomersall, C.D.; Li, T.S.; Antonio, G.; Ko, F.W.; Chan, M.C.; Chan, D.P.; Tong, M.W., et al. Impact of severe acute respiratory syndrome (SARS) on pulmonary function, functional capacity and quality of life in a cohort of survivors. Thorax 2005, 60, 401-409, doi:10.1136/thx.2004.030205.

13. Venkataraman, T.; Frieman, M.B. The role of epidermal growth factor receptor (EGFR) signaling in SARS coronavirus-induced pulmonary fibrosis. Antiviral research 2017, 143, 142-150, doi:10.1016/j.antiviral.2017.03.022.

14. Zhang, P.; Li, J.; Liu, H.; Han, N.; Ju, J.; Kou, Y.; Chen, L.; Jiang, M.; Pan, F.; Zheng, Y., et al. Long-term bone and lung consequences associated with hospital-acquired severe acute respiratory syndrome: a 15-year followup from a prospective cohort study. Bone Research 2020, 8, 8, doi:10.1038/s41413-020-0084-5.

15. Xu, J.; Xu, X.; Jiang, L.; Dua, K.; Hansbro, P.M.; Liu, G. SARS-CoV-2 induces transcriptional signatures in human lung epithelial cells that promote lung fibrosis. Respiratory Research 2020, 21, 182, doi:10.1186/s12931-020-014456.

16. Krewski, D.; Acosta, D., Jr.; Andersen, M.; Anderson, H.; Bailar, J.C., 3rd; Boekelheide, K.; Brent, R.; Charnley, G.; Cheung, V.G.; Green, S., Jr., et al. Toxicity testing in the 21st century: a vision and a strategy. J Toxicol Environ Health B Crit Rev 2010, 13, 51-138, doi:10.1080/10937404.2010.483176.

17. Ankley, G.T.; Bennett, R.S.; Erickson, R.J.; Hoff, D.J.; Hornung, M.W.; Johnson, R.D.; Mount, D.R.; Nichols, J.W.; Russom, C.L.; Schmieder, P.K., et al. Adverse outcome pathways: a conceptual framework to support ecotoxicology research and risk assessment. Environmental toxicology and chemistry 2010, 29, 730-741, doi:10.1002/etc.34.

18. Kramer, V.J.; Etterson, M.A.; Hecker, M.; Murphy, C.A.; Roesijadi, G.; Spade, D.J.; Spromberg, J.A.; Wang, M.; Ankley, G.T. Adverse outcome pathways and ecological risk assessment: bridging to population-level effects. Environmental toxicology and chemistry 2011, 30, 64-76, doi:10.1002/etc.375.

19. AOPwiki. Binding of viral S-glycoprotein to ACE2 receptor leading to acute respiratory distress associated mortality. Availabe online: www. aopwiki.org/aops/320 (accessed on 22 December 2020).

20. Shulla, A.; Heald-Sargent, T.; Subramanya, G.; Zhao, J.; Perlman, S.; Gallagher, T. A Transmembrane Serine Protease Is Linked to the Severe Acute Respiratory Syndrome Coronavirus Receptor and Activates Virus Entry. Journal of Virology 2011, 85, 873-882, doi:10.1128/jvi.02062-10.

21. Tang, T.; Bidon, M.; Jaimes, J.A.; Whittaker, G.R.; Daniel, S. Coronavirus membrane fusion mechanism offers a potential target for antiviral development. Antiviral research 2020, 178, 104792, doi:https://doi.org/10.1016/j.antiviral.2020.104792.

22. Glowacka, I.; Bertram, S.; Müller, M.A.; Allen, P.; Soilleux, E.; Pfefferle, S.; Steffen, I.; Tsegaye, T.S.; He, Y.; Gnirss, K., et al. Evidence that TMPRSS2 Activates the Severe Acute Respiratory Syndrome Coronavirus Spike Protein for Membrane Fusion and Reduces Viral Control by the Humoral Immune Response. Journal of Virology 2011, 85, 4122-4134, doi:10.1128/jvi.02232-10. 
23. Devaux, C.A.; Rolain, J.-M.; Raoult, D. ACE2 receptor polymorphism: Susceptibility to SARS-CoV-2, hypertension, multi-organ failure, and COVID-19 disease outcome. Journal of Microbiology, Immunology and Infection 2020, 53, 425-435, doi:https://doi.org/10.1016/j.jmii.2020.04.015.

24. Catanzaro, M.; Fagiani, F.; Racchi, M.; Corsini, E.; Govoni, S.; Lanni, C. Immune response in COVID-19: addressing a pharmacological challenge by targeting pathways triggered by SARS-CoV-2. Signal Transduction and Targeted Therapy 2020, 5, 84, doi:10.1038/s41392-020-0191-1.

25. Hojyo, S.; Uchida, M.; Tanaka, K.; Hasebe, R.; Tanaka, Y.; Murakami, M.; Hirano, T. How COVID-19 induces cytokine storm with high mortality. Inflammation and Regeneration 2020, 40, 37, doi:10.1186/s41232-020-00146-3.

26. Hariharan, A.; Hakeem, A.R.; Radhakrishnan, S.; Reddy, M.S.; Rela, M. The Role and Therapeutic Potential of NF-kappa-B Pathway in Severe COVID-19 Patients. Inflammopharmacology 2020, 10.1007/s10787-020-00773-9, doi:10.1007/s10787-020-00773-9.

27. Qin, C.; Zhou, L.; Hu, Z.; Zhang, S.; Yang, S.; Tao, Y.; Xie, C.; Ma, K.; Shang, K.; Wang, W., et al. Dysregulation of Immune Response in Patients With Coronavirus 2019 (COVID-19) in Wuhan, China. Clinical Infectious Diseases 2020, 71, 762-768, doi:10.1093/cid/ciaa248.

28. De Abajo, F.J. Renin-angiotensin system inhibitors and COVID-19: overwhelming evidence against an association. The Lancet. Digital health 2020, 10.1016/s2589-7500(20)30294-6, doi:10.1016/s2589-7500(20)30294-6.

29. Bader, M. Tissue Renin-Angiotensin-Aldosterone Systems: Targets for Pharmacological Therapy. Annual Review of Pharmacology and Toxicology 2010, 50, 439-465, doi:10.1146/annurev.pharmtox.010909.105610.

30. Ribeiro-Oliveira, A., Jr.; Nogueira, A.I.; Pereira, R.M.; Boas, W.W.; Dos Santos, R.A.; Simões e Silva, A.C. The renin-angiotensin system and diabetes: an update. Vascular health and risk management 2008, 4, 787-803.

31. Kuba, K.; Imai, Y.; Ohto-Nakanishi, T.; Penninger, J.M. Trilogy of ACE2: a peptidase in the renin-angiotensin system, a SARS receptor, and a partner for amino acid transporters. Pharmacology E therapeutics 2010, 128, 119128, doi:10.1016/j.pharmthera.2010.06.003.

32. Russell, J.A.; Marshall, J.C.; Slutsky, A.; Murthy, S.; Sweet, D.; Lee, T.; Singer, J.; Patrick, D.M.; Du, B.; Peng, Z., et al. Study protocol for a multicentre, prospective cohort study of the association of angiotensin II type 1 receptor blockers on outcomes of coronavirus infection. BMJ Open 2020, 10, e040768, doi:10.1136/bmjopen-2020040768.

33. Gomolak, J.R.; Didion, S.P. Angiotensin II-induced endothelial dysfunction is temporally linked with increases in interleukin-6 and vascular macrophage accumulation. Frontiers in Physiology 2014, 5, doi:10.3389/fphys.2014.00396.

34. Chen, J.; Chen, J.-K.; Nagai, K.; Plieth, D.; Tan, M.; Lee, T.-C.; Threadgill, D.W.; Neilson, E.G.; Harris, R.C. EGFR signaling promotes TGF $\beta$-dependent renal fibrosis. J Am Soc Nephrol 2012, 23, 215-224, doi:10.1681/ASN.2011070645. 
35. Liu, R.-M.; Desai, L.P. Reciprocal regulation of TGF- $\beta$ and reactive oxygen species: A perverse cycle for fibrosis. Redox Biology 2015, 6, 565-577, doi:https://doi.org/10.1016/j.redox.2015.09.009.

36. Tian, H.; Yu, D.; Hu, Y.; Zhang, P.; Yang, Y.; Hu, Q.; Li, M. Angiotensin II upregulates cyclophilin A by enhancing ROS production in rat cardiomyocytes. Mol Med Rep 2018, 18, 4349-4355, doi:10.3892/mmr.2018.9448.

37. Li, A.; Zhang, J.; Zhang, X.; Wang, J.; Wang, S.; Xiao, X.; Wang, R.; Li, P.; Wang, Y. Angiotensin II induces connective tissue growth factor expression in human hepatic stellate cells by a transforming growth factor $\beta$ independent mechanism. Scientific Reports 2017, 7, 7841, doi:10.1038/s41598-017-08334-x.

38. Macconi, D.; Remuzzi, G.; Benigni, A. Key fibrogenic mediators: old players. Renin-angiotensin system. Kidney international supplements 2014, 4, 58-64, doi:10.1038/kisup.2014.11.

39. An, J.; Wei, R.; Zhou, H.; Luong Tiffany, Q.; Gould Michael, K.; Mefford Matthew, T.; Harrison Teresa, N.; Creekmur, B.; Lee, M.S.; Sim John, J., et al. Angiotensin-Converting Enzyme Inhibitors or Angiotensin Receptor Blockers Use and COVID-19 Infection Among 824,650 Patients with Hypertension from a US integrated Healthcare System. Journal of the American Heart Association 2020, 0, e017773, doi:10.1161/JAHA.120.019669.

40. Brojakowska, A.; Narula, J.; Shimony, R.; Bander, J. Clinical Implications of SARS-CoV-2 Interaction With Renin Angiotensin System: JACC Review Topic of the Week. Journal of the American College of Cardiology 2020, 75, 30853095, doi:10.1016/j.jacc.2020.04.028.

41. Guo, T.; Fan, Y.; Chen, M.; Wu, X.; Zhang, L.; He, T.; Wang, H.; Wan, J.; Wang, X.; Lu, Z. Cardiovascular Implications of Fatal Outcomes of Patients With Coronavirus Disease 2019 (COVID-19). JAMA Cardiology 2020, 5, 811-818, doi:10.1001/jamacardio.2020.1017.

42. Robson, B. COVID-19 Coronavirus spike protein analysis for synthetic vaccines, a peptidomimetic antagonist, and therapeutic drugs, and analysis of a proposed achilles' heel conserved region to minimize probability of escape mutations and drug resistance. Comput Biol Med 2020, 121, 103749-103749, doi:10.1016/j.compbiomed.2020.103749.

43. Souza, P.F.N.; Lopes, F.E.S.; Amaral, J.L.; Freitas, C.D.T.; Oliveira, J.T.A. A molecular docking study revealed that synthetic peptides induced conformational changes in the structure of SARS-CoV-2 spike glycoprotein, disrupting the interaction with human ACE2 receptor. International Journal of Biological Macromolecules 2020, 164, 66-76, doi:https://doi.org/10.1016/j.ijbiomac.2020.07.174.

44. Wu, C.; Liu, Y.; Yang, Y.; Zhang, P.; Zhong, W.; Wang, Y.; Wang, Q.; Xu, Y.; Li, M.; Li, X., et al. Analysis of therapeutic targets for SARS-CoV-2 and discovery of potential drugs by computational methods. Acta pharmaceutica Sinica. B 2020, 10, 766-788, doi:10.1016/j.apsb.2020.02.008.

45. Horby, P.; Lim, W.S.; Emberson, J.; Mafham, M.; Bell, J.; Linsell, L.; Staplin, N.; Brightling, C.; Ustianowski, A.; Elmahi, E., et al. Effect of Dexamethasone in Hospitalized Patients with COVID-19: Preliminary Report. medRxiv 2020, 10.1101/2020.06.22.20137273, 2020.2006.2022.20137273, doi:10.1101/2020.06.22.20137273. 
46. Fadaka, A.O.; Sibuyi, N.R.S.; Madiehe, A.M.; Meyer, M. Computational insight of dexamethasone against potential targets of SARS-CoV-2. Journal of Biomolecular Structure and Dynamics 2020, 10.1080/07391102.2020.1819880, 1-11, doi:10.1080/07391102.2020.1819880.

47. Choudhary, S.; Malik, Y.S.; Tomar, S. Identification of SARS-CoV-2 Cell Entry Inhibitors by Drug Repurposing Using in silico Structure-Based Virtual Screening Approach. Frontiers in Immunology 2020, 11, doi:10.3389/fimmu.2020.01664. 